\title{
INCENTIVO E PROMOÇÃO DO ALEITAMENTO MATERNO EM UM HOSPITAL UNIVERSITÁRIO: VIVÊNCIAS DE UM PROJETO DE EXTENSÃO
}

\author{
Anna Priscilla Mendes Alves e Lemes ${ }^{1}$ \\ Jéssica Rodrigues Rosa ${ }^{2}$ \\ João Paulo Assunção Borges ${ }^{3}$ \\ Lori Anisia Martins de Aquino ${ }^{4}$
}

\begin{abstract}
RESUMO: Este trabalho objetiva relatar a experiência de acadêmicas e docentes no projeto de extensão intitulado "Incentivo e promoção do aleitamento materno: trilhando os passos da Iniciativa Hospital Amigo da Criança", do curso de graduação em Enfermagem, vinculado ao "Programa de Extensão Integração Universidade Federal de Uberlândia/Comunidade" (PEIC), realizado em um hospital universitário. Entre dezembro de 2013 e junho de 2014, ocorreram 1.405 partos, predominando a cesárea (66,8\%), na idade entre 19 e 30 anos $(62,1 \%)$. Foram atendidas 401 puérperas, individualmente, e 63 puérperas/acompanhantes em palestras em grupos. Os binômios com dificuldade na amamentação foram acompanhados durante a hospitalização a fim de orientar, auxiliar e incentivar o aleitamento materno. Além disso, 22 profissionais de enfermagem participaram da capacitação, realizada por meio de aula expositiva dialogada. O projeto de extensão evidenciou que a visita diária ao binômio, com avaliação da mamada e orientações às mães, propicia segurança para que elas amamentem seus filhos. Além disso, contribuiu para aumentar os conhecimentos das puérperas/acompanhantes e dos profissionais sobre as vantagens do aleitamento e o manejo clínico da amamentação, e promoveu a vivência e o aprendizado das acadêmicas na realidade profissional.
\end{abstract}

PALAVRAS-CHAVE: Aleitamento materno. Enfermagem neonatal. Saúde da criança.

\section{Incentive and promotion of breastfeeding in a university hospital: experiences of an extension project}

\begin{abstract}
This paper reports the experience of teachers and students in an extension project of the undergraduate Nursing course entitled "Incentivo e promoção do aleitamento materno: trilhando os passos da Iniciativa Hospital Amigo da Criança" ("Encouraging and promoting breastfeeding: tracing the steps of the Amigo da Criança Hospital Iniciative") linked to the "Programa de Extensão Integração Universidade Federal de Uberlândia/Comunidade" (PEIC - Extension Program Federal University of Uberlandia and Community Integration) carried out in a university hospital. Between December/2013 and June/2014, 1405 birth occurred, predominantly via cesarean $(66,8 \%)$, in woman aged between 19 and 30 years $(62,1 \%) .401$ women were attended individually; and 63 carer/women who had recently given birth were attended in groups. Mother and babies with difficulty in breastfeeding were monitored during hospitalization so they could be guided, assisted and encouraged on what concerns breastfeeding. Furthermore, 22 nursing professionals attended the training, which was a dialogued lecture. The extension project showed that the daily visit

\footnotetext{
${ }^{1}$ Graduada em Enfermagem pela Universidade Federal de Uberlândia (annaprix@hotmail.com).

${ }^{2}$ Graduada em Enfermagem na Universidade Federal de Uberlândia (jessikinha_rosa_rodrigues@hotmail.com).

${ }^{3}$ Mestre em Ciências da Saúde pela Universidade Federal de Uberlândia, enfermeiro, professor assistente da Universidade Federal de Uberlândia (enf_joaopaulo@yahoo.com.br).

${ }^{4}$ Mestre em Educação Superior pelo Centro Universitário do Triângulo, professora adjunta da Universidade Federal de Uberlândia (lorianisia@yahoo.com.br).
} 
to the binomials (mother/child), breastfeeding's assessment and guidance to mothers provided them security to breastfeed their children. It contributed to increase the knowledge of the postpartum women/carer and professionals about the benefits of breastfeeding and the clinical management of it. It also promoted the experiencing and learning of the students in professional reality.

KEYWORDS: Breastfeeding. Neonatal nursing. Child health.

\section{INTRODUÇÃO}

A Organização Mundial da Saúde (OMS) e o Fundo das Nações Unidas para a Infância (UNICEF) recomendam que as crianças sejam alimentadas exclusivamente com leite humano durante os seis primeiros meses de vida e que o aleitamento materno (AM) seja continuado até dois anos de idade ou mais (BRASIL, 2009a; OMS, 1993).

No Brasil, em 1981, o Ministério da Saúde criou o "Programa Nacional de Incentivo ao Aleitamento Materno" (PNIAM), almejando aprimorar profissionais de saúde, aconselhar a amamentação individualizada, produzir materiais educativos, estabelecer grupos de apoio à amamentação na comunidade, aprovar leis de proteção à amamentação e controlar o marketing de leites artificiais (SOUZA; ESPÍRITO SANTO; GIUGLIANI, 2008).

De acordo com o Ministério da Saúde, a partir da implementação do PNIAM, o índice de aleitamento materno exclusivo (AME) de crianças com menos de quatro meses cresceu de $35 \%$, em 1999, para 52\% em 2008. Esses índices mostram a conscientização da população sobre a importância do AM exclusivo até os seis meses e os diversos benefícios para mãe e filho (BRASIL, 2009b).

Com o intuito de combater o desmame precoce e contribuir para o crescimento saudável da criança, a OMS e o UNICEF emitiram a Declaração de Innocenti, em 1990, que trata da proteção, da promoção e do apoio ao aleitamento materno (ALMEIDA et al., 2008). Em resposta, a "Iniciativa Hospital Amigo da Criança" (IHAC) foi idealizada pelas duas organizações, naquele ano, para promover, proteger e apoiar o aleitamento materno nas maternidades no momento em que a criança nasce. Nesse contexto, um grupo de especialistas de saúde e nutrição de vários países elaborou um conjunto de medidas denominado "Dez passos para o sucesso do aleitamento materno". O objetivo da IHAC consiste na mobilização de profissionais de saúde e funcionários de hospitais e maternidades para modificar rotinas e condutas, visando prevenir o desmame precoce (LUNARDI; BULHOSA, 2004).

Nesse sentido, uma das exigências para que uma instituição obtenha a certificação de "Hospital Amigo da Criança" é a implantação dos "Dez passos para o sucesso do aleitamento materno", sugeridos pelo UNICEF (2008). São eles:

1. ter uma norma escrita sobre aleitamento materno, que deve ser rotineiramente transmitida a toda a equipe do serviço;

2. treinar toda a equipe, capacitando-a para implementar essa norma;

3. informar todas as gestantes atendidas sobre as vantagens e o manejo da amamentação; 
4. ajudar a mãe a iniciar a amamentação na primeira meia hora após o parto;

5. mostrar às mães como amamentar e como manter a lactação, mesmo se vierem a ser separadas de seus filhos;

6. não dar a recém-rascido nenhum outro alimento ou bebida além do leite materno, a não ser que tenha indicação clínica;

7. praticar o alojamento conjunto: permitir que mães e bebês permaneçam juntos 24 horas por dia;

8. encorajar a amamentação sob livre demanda;

9. não dar bicos artificiais ou chupetas a crianças amamentadas;

10. encorajar o estabelecimento de grupos de apoio à amamentação, para onde as mães devem ser encaminhadas por ocasião da alta hospitalar.

Basicamente, os dez passos consistem em um elenco de medidas destinado a informar sobre os benefícios e o correto manejo do AM a todas as gestantes. As mães devem ser esclarecidas das vantagens do aleitamento e das desvantagens do uso de substitutos do leite materno. Além disso, devem receber noções sobre a lactação e o estímulo para produção do leite, sobre como lidar com as dificuldades e buscar soluções para os problemas que ocorrem durante a amamentação (LAMOUNIER, 1996; PARIZOTO et al., 2009).

Diante da complexidade dessa prática, faz-se necessária a atuação da equipe de saúde em diversas frentes, de forma a integrar todo o serviço e mobilizar todos os profissionais (BECKER, 2001), que devem realizar o acompanhamento e o aconselhamento das nutrizes, adequando suas ações à cultura, aos hábitos, às crenças e à posição socioeconômica das mães (PARADA et al., 2005). A atuação dos profíssionais de saúde pode ter influência positiva ou, por outro lado, negativa no estabelecimento e manutenção do AM, caso não sejam eles capazes de enxergar além do manejo clínico e não ofereçam o suporte necessário às mães (NAKANO et al., 2007).

O projeto de extensão "Incentivo e promoção do aleitamento materno: trilhando os passos da Iniciativa Hospital Amigo da Criança" teve como objetivos promover, proteger, incentivar e apoiar o AM, por meio da implantação dos "Dez passos para o sucesso da amamentação", preconizados pelo UNICEF. Especificamente, objetivou praticar ações educativas de incentivo e promoção do AM, por meio de orientações às gestantes, às puérperas nutrizes, aos acompanhantes e à equipe multiprofissional dos serviços de Ginecologia e Obstetrícia, alojamento conjunto (AC) e berçário do Hospital de Clínicas da Universidade Federal de Uberlândia (HC-UFU). Além disso, a execução do projeto buscou inserir os acadêmicos do curso de Enfermagem na rotina desses serviços.

A partir da realização deste estudo, objetivou-se relatar a experiência vivenciada por acadêmicas e docentes de Enfermagem no período de realização de um projeto de extensão em um hospital universitário da cidade de Uberlândia, Minas Gerais. 


\section{METODOLOGIA}

Este trabalho trata-se de estudo descritivo, do tipo relato de experiência, acerca da vivência da equipe composta por discentes e docentes do projeto de extensão intitulado "Incentivo e promoção do aleitamento materno: trilhando os passos da Iniciativa Hospital Amigo da Criança" do curso de graduação em Enfermagem da Universidade Federal de Uberlândia, vinculado ao Programa de Extensão Integração UFU/Comunidade (PEIC).

O PEIC tem o objetivo de incentivar e apoiar projetos que contribuam para reafirmar a extensão como processo acadêmico definido e efetivado em função das exigências da realidade, indispensável na formação do aluno, na qualificação docente e no intercâmbio com a sociedade, além de oferecer respostas às necessidades da sociedade, por meio de ações extensionistas relacionadas às áreas temáticas definidas pela Política Nacional de Extensão Universitária (UNIVERSIDADE, 2009).

A equipe executora do projeto foi composta por duas acadêmicas do curso de Enfermagem da UFU e por dois enfermeiros docentes das disciplinas "Atenção Integral à Saúde da Criança I" e "Atenção Integral à Saúde da Criança II" do curso de Enfermagem da UFU. As atividades do projeto aconteceram durante o período de agosto de 2013 a julho de 2014 no alojamento conjunto de um hospital universitário da cidade de Uberlândia-MG. A carga horária semanal de atividades das acadêmicas bolsistas foi de 20 horas e foram realizadas ações extensionistas, com foco na educação em saúde de puérperas e seus familiares, com vistas ao incentivo e promoção do AM, por meio de orientações individuais e coletivas, distribuição de material informativo e capacitação da equipe de Enfermagem quanto ao AM.

Foi aplicado o "Instrumento de admissão e evolução da parturiente no pré-parto, parto e puerpério", que reúne dados referentes a: acompanhamento pré-natal, momento do parto e nascimento, como o tipo de parto; adaptação do recém-nascido; início do AM na primeira hora de vida; bem como as ações de promoção do AM implementadas durante a permanência do binômio mãe-recém-nascido no alojamento conjunto até a alta hospitalar.

Às puérperas/nutrizes internadas no alojamento conjunto, também foi aplicado o "Formulário de Avaliação da Mamada", que estabelece indicadores baseados nos sinais de que a amamentação está adequada e nos sinais de dificuldade durante a mamada. Também foram realizadas orientações relativas à importância da amamentação, ao posicionamento da mãe e do bebê durante as mamadas, aos cuidados com as mamas, entre outros. Cada puérpera/nutriz atendida recebeu um folder explicativo, produzido pelas acadêmicas, com supervisão dos docentes envolvidos, contendo informações básicas referentes às vantagens do AM e orientações para uma amamentação bem sucedida.

\section{RESULTADOS E DISCUSSÃO}

A fase inicial do projeto, de agosto a novembro de 2013, foi destinada à imersão das duas acadêmicas e dos dois docentes no estudo de temas relacionados ao AM. Foi uma etapa constituída de exaustiva revisão bibliográfica, participação em grupos semanais de estudo, discussão de artigos científicos, além da elaboração do material didático, do tipo folders 
explicativos e apresentações em Power Point, direcionados às puérperas/nutrizes e à capacitação/atualização da equipe de Enfermagem.

No período de dezembro de 2013 a junho de 2014, segundo informações do Setor de Estatísticas e Informações Hospitalares, foram registrados 1.405 partos, dos quais 938 $(66,8 \%)$ foram cesáreas e 467 (33,2\%) partos normais. Desse total de mulheres, $1282(91,2 \%)$ eram de Uberlândia, e apenas $123(8,8 \%)$, de outras cidades. A faixa etária que se destacou foi a de 19 a 30 anos, representando $872(62,1 \%)$ mulheres.

Durante as atividades do projeto e em cumprimento às atividades previstas em escala semanal, as acadêmicas prestaram atendimento individualizado a $401(28,5 \%)$ puérperas, que foram aleatoriamente abordadas enquanto permaneceram internadas no AC. Esse atendimento englobou a aplicação do "Instrumento de admissão e evolução da parturiente no pré-parto, parto e puerpério" e do "Formulário de avaliação das mamadas", além do fornecimento de informações e orientações quanto ao manejo adequado do AM, entre outros.

De acordo com Weiderpass et al. (1998), as mães que se submetem à cesariana eletiva, quando comparadas àquelas que tiveram partos normais, apresentam um risco aumentado de não iniciar a amamentação ou de interrupção completa da lactação no primeiro mês de vida. É possível que essa variação ocorra devido ao padrão de atendimento pós-operatório de alguns hospitais, que dificulta o alojamento conjunto, o aleitamento à livre demanda e, ainda, introduzem precocemente outros alimentos na dieta do recém-nascido.

Houve também uma quantidade expressiva de mulheres com idade entre 14 e 18 anos, totalizando 170 (12,1\%). Segundo o Ministério da Saúde, a proporção de mães adolescentes vem diminuindo no Brasil, porém ainda é expressiva, com 20,4\% no ano de 2008. Na região Sudeste, ocorreu uma redução de 20,4\%, no ano de 1997 , para $17 \%$, em 2008. Além disso, notou-se um aumento da proporção de mães com mais de 35 anos de idade, de 8,1\%, no ano de 1997, para 9,9\%, em 2008 (BRASIL, 2011).

Ao comparar os índices de partos em adolescentes obtidos na instituição em que foi desenvolvido o projeto com os índices nacionais supracitados, evidencia-se que o percentual de mães adolescentes encontra-se abaixo tanto do índice nacional como do correspondente à região Sudeste, no ano de 2008.

Para Araújo et al. (2008), a idade da mãe influencia na duração do AM, sendo que as mais jovens amamentam por períodos mais curtos. Os fatores que podem provocar o desmame precoce são: nível educacional mais baixo, o estado civil solteira, menor poder aquisitivo, insegurança e falta de apoio das próprias mães ou familiares próximos.

Durante o desenvolvimento do projeto, os atendimentos ocorreram de forma individualizada, a cada puérpera/nutriz, enquanto estavam internadas no alojamento conjunto. Os binômios mãe-filho que, durante a avaliação da mamada, apresentaram algum sinal de dificuldade, foram acompanhados pelas acadêmicas por meio de visitas regulares e consecutivas durante a internação hospitalar. Dentre as 401 puérperas que foram abordadas individualmente pelas acadêmicas durante as atividades do projeto, apurou-se que $264(65,8 \%)$ foram submetidas ao parto cesárea e 137 (34,2\%), ao parto normal. 
Além dos atendimentos individuais supracitados, também foram orientadas outras 63 puérperas e seus acompanhantes, por meio de oito palestras em grupo, realizadas esporadicamente pelas acadêmicas, na sala de espera do setor. O tema central compreendeu orientações técnicas sobre o AM, como composição do leite materno, vantagens e benefícios para a mãe e o recém-nascido, aleitamento até o sexto mês, posições e técnicas para amamentar, possíveis dificuldades da amamentação, entre outros assuntos.

Foi elaborada uma aula acerca do tema "Posicionamento do binômio mãe-RN durante a mamada e os sinais da pega correta", com duração média de 30 minutos de apresentação do tipo expositiva dialogada, para capacitação e atualização da equipe de Enfermagem do Serviço de Ginecologia e Obstetrícia e Alojamento Conjunto do HC-UFU. A aula foi ministrada em quatro momentos diferentes, contemplando todos os turnos de trabalho, para atingir o maior número possível de profissionais de Enfermagem. Participaram 22 profissionais, entre eles enfermeiras, técnicas e auxiliares de Enfermagem, o que correspondeu a $61,1 \%$ da equipe de Enfermagem.

Dentre os principais obstáculos encontrados na execução do projeto, podemos destacar a dificuldade em abordar algumas puérperas, devido ao cansaço inerente ao período pós-parto e à presença de acompanhantes e visitantes no momento da abordagem, o que dificultava a conversa e a avaliação da mamada.

Por meio do projeto de extensão, as acadêmicas puderam aprimorar seus conhecimentos a respeito do AM e identificar, na prática, as principais dificuldades e dúvidas das mães durante o aleitamento materno exclusivo, podendo, assim, orientar e intervir no momento em que o aleitamento começa a ser estabelecido. Participar do projeto também contribuiu para a experiência profissional e satisfação pessoal das acadêmicas.

Do ponto de vista da atuação docente, foi possível promover um ambiente favorável à aplicação de conhecimentos acerca do incentivo, da promoção e da proteção do AM na unidade do alojamento conjunto, por meio das atividades executadas pelas acadêmicas com a supervisão e o suporte dos docentes. A realização de reuniões de estudo, com discussão de artigos científicos sobre o tema, proporcionou espaços de reflexão e crítica das práticas adotadas frente ao AM.

A atividade de extensão promoveu a troca de experiências, vivências e conhecimentos entre docentes, discentes e comunidade, favorecendo o aperfeiçoamento da equipe envolvida no tema abordado, além de contribuir com as estratégias de educação em saúde da população atendida.

\section{CONSIDERAÇÕES FINAIS}

As atividades desenvolvidas no projeto de extensão promoveram a vivência prática do manejo do AM, proporcionando o aprendizado em relação à amamentação e às dificuldades apresentadas pelas puérperas/nutrizes. O AM é um ato que requer contínuo aprendizado, seja pela equipe de saúde ou pelas mães que almejam amamentar seu bebê. Muitas vezes, é preciso que os profissionais de saúde auxiliem as mães a estabelecerem a amamentação, orientandoas, apoiando-as e incentivando-as durante todo o processo. 
A realização do projeto, por meio do acompanhamento diário e da avaliação profissional e acadêmica da evolução do AM, permitiu identificar que os binômios atendidos apresentavam maior segurança no estabelecimento da amamentação. A avaliação da mamada e a oferta de orientações e apoio às mães contribuíram para aumentar o seus conhecimentos sobre as vantagens do leite materno, a posição adequada do binômio e a pega correta no momento da mamada, entre outros.

As atividades de extensão são fundamentais no processo de fortalecimento da formação acadêmica, intimamente relacionada com as questões de demanda social, permitindo que a Universidade se torne um espaço de produção de conhecimento voltado para as necessidades da população em geral, em especial, dos grupos mais vulneráveis.

REFERÊNCIAS

ALMEIDA, G. G. et al. Proteção, promoção e apoio ao aleitamento materno em um hospital universitário. Ciência \& Saúde Coletiva, Rio de Janeiro, v. 13, n. 2, p. 487-494, 2008.

ARAÚJO, O. D. et al. Aleitamento materno: fatores que levam ao desmame precoce. Revista Brasileira de Enfermagem, Brasília, v. 61, n. 4, p. 488-492, 2008.

BRASIL. Ministério da Saúde. Atenção à saúde do recém-nascido: guia para os profissionais de saúde: cuidados gerais. Brasília, DF: Ministério da Saúde, 2011.

. Ministério da Saúde. Mais saúde: direito de todos. 2009b. Disponível em: $<$ http://bvsms.saude.gov.br/bvs/pacsaude/not_03082009.php>. Acesso em: 22 mar. 2013.

- Ministério da Saúde. Saúde da criança: nutrição infantil: aleitamento materno e alimentação complementar. Brasília: Editora do Ministério da Saúde, 2009a.

BECKER, D. No seio da família: amamentação e promoção da saúde no programa de saúde da família. 2001. 127f. Dissertação (Mestrado) - Fundação Oswaldo Cruz, Escola Nacional de Saúde Pública, Rio de Janeiro, 2001.

LAMOUNIER, J. A. Promoção e incentivo ao aleitamento materno: Iniciativa Hospital Amigo da Criança. Jornal de Pediatria, Rio de Janeiro, v. 72, n. 6, p. 363-368, 1996.

LUNARDI, V. L.; BULHOSA, M. S. A influência da iniciativa hospital amigo da criança na amamentação. Revista Brasileira de Enfermagem, Brasília, v. 57, n. 6, p. 683-6, nov./dez. 2004.

NAKANO, A. M. et. al. O espaço social das mulheres e a referência para o cuidado na prática da amamentação. Revista Latino-Americana de Enfermagem, Ribeirão Preto, v. 15, n. 2, p. 230-238, mar./abr. 2007.

PARADA, C. M. G. de L. et al. Situação do aleitamento materno em população assistida pelo programa de saúde da família. Revista Latino-Americana de Enfermagem, Ribeirão Preto, v. 13, n. 3, p. 407-414, maio/jun. 2005.

PARIZOTO, G. M. et al. Tendência e determinantes do aleitamento materno exclusivo em crianças menores de 6 meses. Jornal de Pediatria, Rio de Janeiro, v. 85, n. 3, p. 201-208, 2009. 
SOUZA, C. B.; ESPÍRITO SANTO, L. C.; GIUGLIANI, E. R. J. Políticas públicas de incentivo ao aleitamento materno: a experiência do Brasil. 2008. Disponível em: $<$ http://portal.saude.gov.br/portal/arquivos/pdf/artigo_franca_novo.pdf $>$. Acesso em: 22 mar. 2013.

UNICEF - Fundo das Nações Unidas para a Infância e Adolescência. Manejo e promoção do aleitamento materno num hospital amigo da criança. Manual Técnico. Genebra: OMS/UNICEF, 1993.

Dez passos para o sucesso do aleitamento materno. 2008. Disponível em: <http://www.unicef.org/brazil/pt/activities_9999.htm>. Acesso em: 19 mar. 2013.

UNIVERSIDADE FEDERAL DE UBERLÂNDIA. Pró-reitoria de extensão, cultura e assuntos estudantis. 2009. Disponível em: <http://www.proex.ufu.br/peic-programaextens $\% \mathrm{C} 3 \%$ A3o-integra $\% \mathrm{C} 3 \% \mathrm{~A} 7 \% \mathrm{C} 3 \% \mathrm{~A} 30$-ufucomunidade $>$. Acesso em: 23 ago. 2014.

WEIDERPASS, E. et al. Incidência e duração da amamentação conforme o tipo de parto: estudo longitudinal no Sul do Brasil. Revista de Saúde Pública, São Paulo, v.32, n.3, p. 225$231,1998$.

Submetido em 28 de agosto de 2014.

Aprovado em 17 de setembro de 2014. 